[22] V. Degiorgio, "On the properties of the spectral bleaching in saturable dyes," Appl. Phys. Letters, vol. 10, pp. 175-177, March 15, 1967.

[23] W. Kaplan, Ordinary Differential Equations. Reading, Mass.: Addison-Wesley, 1960 , ch. 6.

[24] W. G. Wagner and B. A. Lengyel, "Evolution of the giant pulse in a laser," $J$. Appl. Phys., vol. 34, pp. 2040-2046, July 1963.

[25] J. Villar and L. Lindquist, "Spectral absorption and decay, kinetics of the triplet state of phthalocyanine in solution," Compt. Rend. Acad. Sci. (Paris), vol. B264, pp. 1807-1810, June 26, 1967.

[26] V. Daneu, C. A. Sacchi, and O. Svelto, "Single transverse and longitudinal mode $Q$-switched ruby laser," IEEE $J$.
Quantum Electronics, vol. QE-2, pp. 290-293, August 1966.

[27] M. Hercher, "Single mode operation of a $Q$-switched ruby laser," Appl. Phys. Letlers, vol. 7, pp. 39-41, July 1965.

[28] W. West, S. Pearce, and F. Grum, "Stereoisomerism in cyanine dyes-meso-substituted thiacarbocyanines," $J$. Phys. Chem., vol. 71 , pp. 1316-1326, April 1967.

[29] A. V. Buettner, "Radiationless transitions in cyanine dyes," J. Chem. Phys., vol. 46, pp. 1398-1401, February 15, 1967.

[30] M. L. Spaeth and D. P. Bortfeld, "Stimulated emission from polymethine dyes," Appl. Phys. Letters, vol. 9, pp. 179-181, September 1, 1966.

[31] P. J. McCartin, "Observation of metastable geometrical isomers of cyanines by flash photolysis," J. Chem. Phys., vol. 42, pp. 2980-2981, April 15, 1965.

\title{
Correspondence
}

\section{A Method for the Control of Gas Pressure in the Xenon Laser}

In order to facilitate measurements of the behavior of a xenon laser over a range of gas pressures, we have developed a method for controlling the pressure in a sealed-off tube.

If a tube is initially filled to the desired pressure (a few mtorr), absorption of xenon during operation results in rapid pressure reduction [1]. Clark and Hubach reported the maintenance of a constant pressure by overfilling the tube and reducing the xenon vapor pressure by condensing it on a surface cooled with liquid nitrogen [2]. The method here presented employs a variable-temperature surface, which is cooled with liquid nitrogen, but partially insulated from it.

Fig. 1 shows the construction of the device. A glass bulb attached to the laser tube has a folded continuous conductive strip bonded to its surface, which serves as a heating element. Leads from a power supply are attached by means of clamps on the bulb neck. The bulb is insulated with cotton gauze and a surrounding glass envelope,

Manuscript received June 27, 1968; revised August 15, 1968.

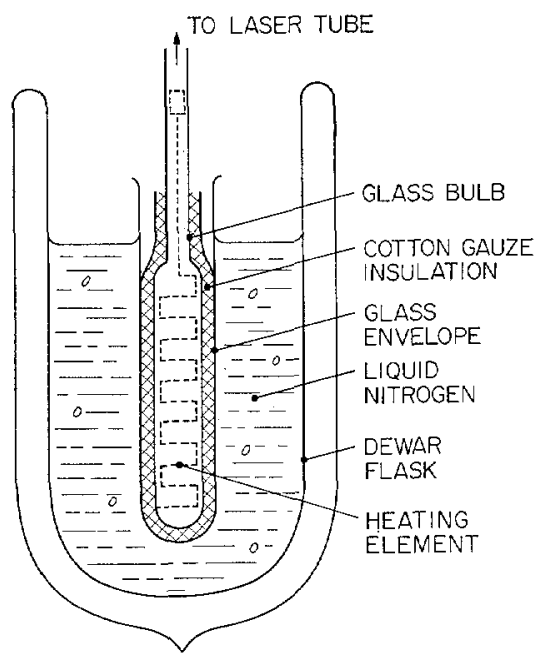

Fig. 1. Variable-temperature cold bulb assembly. and the assembly is immersed in a dewar of liquid nitrogen. It is nesessary to plug the mouth of the dewar to prevent condensation of moisture in the insulation.

The pressure of gas in the laser is controlled by the power supplied to the heating element. For a particular assembly, the equilibrium

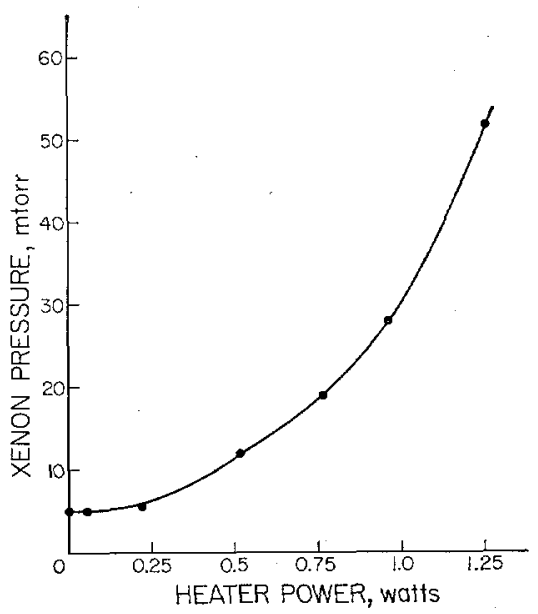

Fig. 2. Xenon pressure versus heater power to cold bulb.

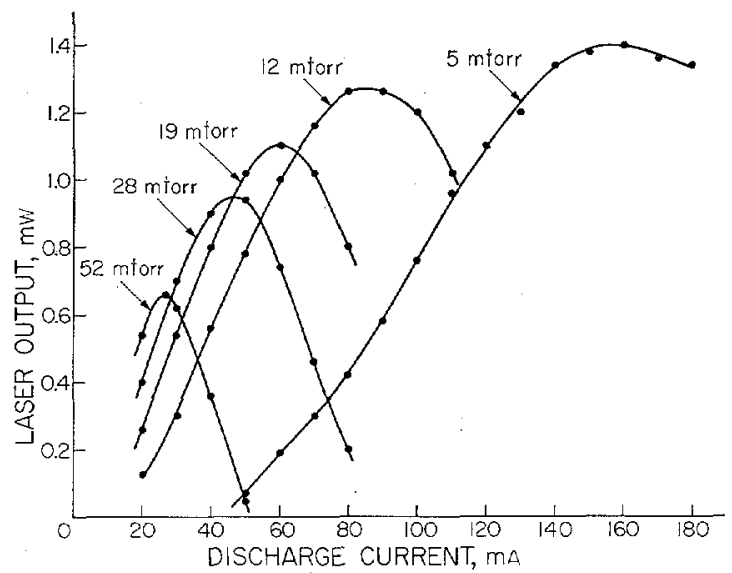

Fig. 3. 3.5- $\mu$ laser output versus discharge current and gas pressure. 
temperature at a given power input depends upon the characteristics of several heat conduction paths, so the design is largely empirical. A typical curve of pressure versus power input is shown in Fig. 2. Provided that the conductivity of the outer envelope is sufficiently higher than that of the gauze insulation, the equilibrium temperature does not vary significantly with the liquid nitrogen level, so the pressure may be kept constant with occasional refilling of the dewar.

As an illustration of the utility of the method, curves of power output versus discharge current and gas pressure for an experimental laser are presented in Fig. 3.

\section{ACKNOWLEDGMEN'T}

Appreciation is due to Hughes Research Laboratories, Malibu, Calif., for supplying a laser tube that was modified for this work, and to Professor Amnon Yariv for helpful comments and suggestions.

Desmond R. Armstrong Dept. of Elec. Engrg. California Institute of Technology Pasadena, Calif.

\section{REFERENCES}

[1] P. 0. Clark, "Investigation of the operating characteristics of the $3.5 \mu$ xenon laser," IEEE J. Quantum Electronics; vol. QE-1, pp. 109-113, June 1965 . 2] Clark and Hubach,

\section{Measurement of the Beam Profile of a $\mathrm{CO}_{2}$ Laser}

Abstract-Foamed polystyrene is used to form a permanent recording of infrared laser beams, from which the intensity distribution can be calculated quantitatively. Minimum-power densities of $450 \mathrm{~mW} / \mathrm{cm}^{2}$ can be recorded. This technique is used to analyze the single-mode operation of a $\mathrm{CO}_{2}$ laser.

It is often desirable to have a quick determination of the beam profile of an infrared laser beam by producing a visible pattern of the intensity distribution of the light. This is important for alignment purposes and for determination of the oscillating modes. By measuring the quantitative power distribution the purity of the modes and the divergence of the beam may be calculated. From these the optical perfection of the cavity can be inferred. The beam profile also determines the size of a focused spot and its energy distribution, which is important when studying the interaction between highpower-density beams and materials.

At very high power densities (approx. $10 \mathrm{~W} / \mathrm{cm}^{2}$ ) the beam can be displayed by heating a refractory target material to incandescence. At lower power densities thermographic screens have been used [1], [2]. These are ultraviolet phosphors that can be quenched thermally. They can be made with sensitivities as high as $1 \mathrm{~mW} / \mathrm{cm}^{2}$ [1], and with fast ( $\frac{1}{10}$ second) time constant and high resolution at lower sensitivities [2]. They are of high contrast and very useful for alignment but do not lend themselves to quantitative measurements. Permanent records can be made by thermally developing UV exposed Kalvar films [3]. Integrated exposures of $0.2 \mathrm{~W} \cdot \mathrm{s} / \mathrm{cm}^{2}$ are required for development. Continuous tone images can be obtained, but contrast and slope of the H-D curve vary with exposure parameters, so that quantitative results cannot be obtained.

In the absence of a continuous tone recording material with known characteristics, quantitative measurements can be made with a material of well-defined threshold energy density. A number of exposures are made with different amounts of total energy $Q_{n}$ of the same laser beam. The energy is varied either by varying the exposure time at constant power or by attenuating the power with a suitable nondistorting attenuator. The corresponding energy

Manuscript received July 19, 1968; revised August 23, 1968. density is $q_{n}(\mathbf{r})$ and

$$
Q_{n}=\int q_{n}(\mathbf{r}) d \mathbf{r}
$$

On the recording material there will be a curve $\mathrm{r}_{n}\left(Q_{n}\right)$ at which the energy density is just equal to the threshold energy density of the materials $q_{\text {th }}$, so that

$$
q_{n}\left(\mathbf{r}_{n}\right)=q_{\mathrm{th}} .
$$

The normalized shape of the energy (or power) distribution of the laser beam is given by

$$
\frac{q_{n}(\mathbf{r})}{Q_{n}}=\frac{q_{\mathrm{th}}}{Q_{n}} \quad\left(\mathbf{r}=\mathbf{r}_{n}\right) .
$$

A plot of $\left(Q_{n}\right)^{-1}$ vs. $\mathbf{I}_{n}$ then depicts the normalized distribution divided by the threshold energy density.

We have used foamed polystyrene as a sensitive recording material with very sharp threshold. Thresholds have been found to vary no more than 20 percent from sample to sample, Resolution is limited by the size of the bubbles in the material. Because of the low thermal conductivity exposure times up to 10 seconds can be used, with complete integration of the power.

Typical results are shown in Fig. 1. A flowing gas $\mathrm{CO}_{2}-\mathrm{N}_{2}-\mathrm{He}$ laser was used with 186-cm-long 2.5-cm-diameter discharge tube and external mirrors. The Fabry-Perot cavity was $300 \mathrm{~cm}$ long with a flat, partially transmitting output mirror and a totally reflecting concave mirror of $10^{3} \mathrm{~cm}$ radius of curvature. The mode structure was controlled by a circular aperture in the cavity and by varying the gain of the tube. The exposures shown in Fig. 1 were taken at a distance of $700 \mathrm{~cm}$ from the output mirror. The first set was taken with the smallest aperture at which lasing would take place $(0.9-\mathrm{cm}$ diameter) so that a pure $\operatorname{TEM}(00)$ mode is produced. The second set appears at a larger aperture and only for certain values of gain. It represents an almost degenerate TEM $(0 n)^{*}$ mode.

Because both beam configurations exhibit circular symmetry it is sufficient to plot the power as a function of radius. Fig. 2 shows

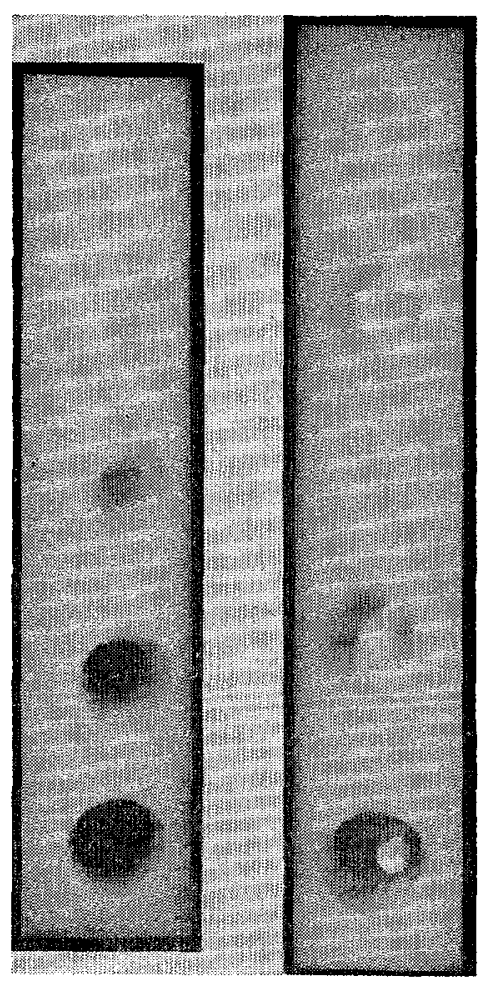

Fig. 1. Recordings of the beam profile of a $\mathrm{CO}_{2}$ laser on foamed polystyrene. Two typical sets of exposures are shown for two different beam modes. The beam energy is varied by using exposure times from $1 / 2$ to 10 seconds. 